\title{
Geht es
Ihnen gut?
}

Selbstfürsorge aus hypnosystemischer Sicht Ergotherapeuten ist es wichtig, dass es ihren Klienten gut geht. Selbst kommen sie manchmal kaum dazu, ausreichend zu trinken oder erholsame Pausen zu verbringen. Falls es Ihnen auch so geht und Sie gerne besser auf sich achtgeben möchten, hat Karin Probst ein paar Tipps für Sie.

Hand aufs Herz: Wann waren Sie das letzte Mal so richtig faul? Wann haben Sie sich Zeit genommen, stundenlang mit Ihrem Lieblingsbuch im Bett zu liegen? Und wann hatten Sie das letzte Mal ein schlechtes Gewissen à la „Ich sollte unbedingt mehr Sport machen“ oder „Ich kann doch jetzt nicht so egoistisch sein und die Vertretung absagen“?

Wenn Ihnen eher die Druckgefühle auf der Brust vertraut sind, das Verschieben der Dinge, die Ihnen eigentlich guttäten - dann ist der folgende Artikel für Sie vielleicht ganz interessant. Und wenn Sie zu den Glückseligen gehören, die das Nichtstun genießen können, dann lesen Sie auf keinen Fall weiter, sondern nutzen die Zeit, um sich etwas Gutes zu tun.

Therapeuten geben viel, nehmen wenig $\rightarrow$ Viele Menschen kennen das: Man weiß eigentlich genau, was einem guttäte - man kommt nur so selten dazu. Und wenn man sich dann endlich doch mal eine kleine Auszeit gönnt, dann plagt einen das schlechte Gewissen oder das Grübeln über die Belange der Arbeit.

Warum fällt es uns so schwer, im Moment sein zu können und die Balance von Egoismus und Altruismus mit Leichtigkeit zu finden? Warum tun sich gerade Menschen in heilenden, therapeutischen und pflegenden Berufen so schwer, für sich selbst zu sorgen? Diese Berufsgruppen sind so achtsam im Geben und versagen sich oft selbst schon die Erfüllung der eigenen Grundbedürfnisse. Oder trin- 


\section{Übung}

\section{Minuten Innehalten}

Im Zen-Kloster ist es ein Ritual, beim

Klang eines Glöckchens kurz innezuhalten

und zu reflektieren: Ergibt das, was ich

im Moment mache, überhaupt Sinn?

Fühlt es sich stimmig an? Was brauche

ich eigentlich? Es reichen drei Minuten

am Tag - ein kleines Innehalten, um ein

freundliches Im-Moment-Sein zu üben.

Vielleicht können Ihnen hierbei die

folgenden Fragen hilfreich sein:

$\rightarrow$ im Moment sein:

Was genau ist jetzt in mir? Nicht

morgen, nicht gestern, nicht bei

anderen - sondern jetzt und in mir.

$\rightarrow$ Achtsamkeit für Bedürfnisse:

Was brauche ich eigentlich gerade?

Welches Bedürfnis möchte gehört und

erfüllt sein? Was mögen die Bedürf-

nisse meines Gegenübers wohl sein?

$\rightarrow$ Es ist, was es ist:

Was ist meine Konstruktion der

Wirklichkeit? Ist meine Bewertung

über die Situation wirklich echt?

$\rightarrow$ Verantwortung:

Was ist langfristig zum Wohle aller

jetzt wohl das Beste?

$\rightarrow$ Worst Case:

Wie könnte ich sicherstellen, dass das

Schlimmstmögliche eintrifft?

(Das ist eine paradoxe Intervention -

und manchmal lautet die Antwort:

Einfach so weitermachen wie bisher.)

$\rightarrow$ VW-Regel:

Welchen Wunsch kann ich statt

einem Vorwurf aussprechen?

$\rightarrow$ Wertschätzung:

Was von dem, was ist, kann ich als die

jetzt beste Lösung wertschätzen?

ken Sie zum Beispiel ausreichend Wasser? Nehmen Sie sich schöne, ruhige Pausen im Alltag? Lassen Sie sich täglich verwöhnen? Pflegen und hegen Sie Ihren Körper mit liebevollen Bemerkungen? Oder gehören auch Sie zu der Spezies, die sich bei jedem Blick in den Spiegel mit Selbstabwertungen belegt wie: „Mensch, ich sollte dringend abnehmen!“ Vielleicht gelingt es Ihnen aber auch, bereits die Form Ihres Nasenrückens oder Ihre Augen mit Begeisterung zu bewundern.

Wir können es nicht jedem recht machen $\rightarrow$

Gerade unter Therapeuten finde ich viele Menschen, die es gerne haben, wenn es anderen gut geht, wenn die Arbeit mit Qualität gemacht ist und man es allen recht machen kann. Das ist an sich etwas Feines, doch nur selten ist dabei auch inkludiert, es sich selbst recht zu machen. Im Grunde suchen wir nach einer harmonischen Lösung, die für alle gut ist. Es auszuhalten und angemessen zu bedauern, dass man noch nicht Superwoman ist, scheint manchmal schwerer zu sein, als mit schlechtem Gewissen oder Schuldgefühlen hin und her zu grübeln.

Wir brauchen eine Art Ambivalenz-Management. Es also auszuhalten, dass wir trotz aller Mühen langfristig kaum schaffen können, die Belange der Arbeit, Familie und Selbstfürsorge harmonisch und gerecht auszubalancieren. Viele Menschen in meinen Seminaren sagen, sie könnten nicht nein sagen und möchten dies dringend lernen. Doch aus meiner Sicht stimmt das so nicht ganz, weil sie bereits nein sagen können, und zwar zu sich selbst. Es gilt also zu lernen, auch ein Nein zu einem anderen zu sagen und ein Ja zu sich.

\section{Man weiß eigentlich} genau, was einem guttäte - man kommt nur so selten dazu.

\section{Innehalten und über-}

legen $\rightarrow$ Nehmen wir als Beispiel die Situation, dass mich eine Kollegin um Unterstützung bittet - obwohl ich selbst dringend meine Arbeit fertig machen muss. Was tun? Meist entscheiden wir uns für die Belange der Kollegin, weil Altruismus sozusagen in unseren Genen verankert ist und wir oft zugunsten kurzfristiger Lösungen entscheiden. Nach dem Motto: Dann mache ich es halt, dann habe ich meine Ruhe. Evolutionär gesehen hatte die Priorisierung von kurzfristig wirksamen Lösungen Sinn, weil man schließlich nicht wusste, wie lange man noch leben wird. Heutzutage kann es hilfreich sein, zwischen den vielen Anforderungen, die von außen und innen auf Erledigung drängen, kurz innezuhalten und sich zu überlegen: Was ist langfristig gesehen meinen Zielen dienlicher? Ist es wirklich besser, mit Unmut dem Drängen der Kollegin nachzugeben und eigene Belange zurückzustellen?

Leider haben wir in der Entwicklung unserer Spezies bisher keine evolutionären Vorteile von Sowohl-als-auch-Lösungen generieren können: Sowohl deine Belange sind wichtig als möglich ist. auch meine. Wenn man also das tägliche Druckerleben als Ausdruck dessen sehen könnte, dass im Moment zwei oder manchmal noch weitere Bedürfnisse in uns lebendig sind, dann könnten wir vielleicht anfangen, uns klar und konsequent für ein Nacheinander zu entscheiden: „Ich mache jetzt erst meine Arbeit fertig, und dann helfe ich dir gerne noch 20 Minuten." Oder mit einem Bedauern das Ansinnen ablehnen: „Liebe Kollegin, ich würde dich sehr gerne unterstützen, weiß aber gerade nicht wie, weil ich bis zum Feierabend noch so viel zu tun habe, dass ich froh bin, wenn ich das schaffen kann. Was machen wir denn jetzt?“

\section{Mit systemischer Hypnotechnik das Gehirn} austricksen $\rightarrow$ Hilfreich in solchen Momenten können Erkenntnisse aus der systemischen Hypnotechnik sein, die Gunther Schmidt vom Milton-Erickson-Institut in Heidelberg entwickelt hat. Seine Vorträge auf YouTube demonstrieren sehr deutlich die Wirksamkeit davon, gesteuert wird, worauf wir unsere Aufmerksamkeit richten ( $\odot$ INTERNET, S. 42). Wenn wir also den ganzen Tag vor uns hinmurmeln: „Ich habe keine Zeit“, dann glaubt unser Gehirn uns das. Hier könnte man bereits von einer Hypnose sprechen - nur leider von keiner dienlichen. Viel dienlicher wäre es, zu sagen: „Ich habe Zeit. Und alles ist meine Zeit, die ich mir schenke oder einer Anforderung widme, wenn ich es möchte." Vielleicht können Sie den Unterschied bereits spüren, wenn Sie sich beide Formulierungen mal sagen und darauf achten, ob und wo ein Druckerleben in Ihrem Körper stattfindet. „Ich habe keine Zeit“ oder „Ich habe Zeit, und alles ist meine Zeit“. Was fühlt sich besser an? Beides ist wahr und hat nichts mit think positive oder rosa gefärbter Duftlampenkommunikation zu tun. Es kann aber dabei helfen, selbst sehr herausfordernde Themen so anzusprechen, dass eine Lösung

Wie würde es sich anfühlen, wenn Sie zu Ihrer Chefin sagen: „Ich würde mich sehr freuen, wenn Sie mir drei Punkte nennen könnten, die Sie an meiner Arbeit schätzen"? Anstatt zu sagen: „Sie können auch nur meckern.“ Da unser Gehirn kaum in der Lage ist, Annahmen 
und Wirklichkeit zu unterscheiden, ist die erste Variante viel dienlicher, weil hier bereits der Lösungsvorschlag impliziert ist. Die Kritik muss erst gar nicht formuliert werden, sondern lediglich eine Hypnotrance auf die Lösung. Und das ist somit dienlicher, weil man den anderen nicht durch Kritik beschämt, sondern einfach sagt, was man will. Für unseren Einkauf beim Metzger haben wir dieses zielführende Verhalten schon gelernt. Hier treffe ich kaum Menschen, die sagen: „Ich möchte keine Salami“,

Ihnen gestalterische Kraft zu nehmen und Sie und Ihre Nachkommen zu schädigen.

Sagen Sie in einer Abgrenzungssituation zu sich: „Ich mache es jetzt, so gut ich kann. Wenn ich es besser könnte, dann würde ich es so machen. Ich darf meine Belange genauso im Blick haben wie die Belange von außen. “ Damit helfen Sie Ihrem Gehirn, mit den Bereichen zu antworten, die Denken und wohlüberlegte Entscheidungen möglich machen - anstatt mit gestressten Reflexhandlungen, Schuldzuweiund davon ausgehen, dass der Metzger dann schon weiß, dass man feine Gelbwurst möchte.
Statt sich zu ärgern, lieber klar ansprechen, was man als hilfreich empfinden wirrde. sungen, Druckgefühlen oder Abwertungen.

Täglich Wertschätzung trainieren $\rightarrow$ Karl Valentin sagte einmal: „Ich freue mich, wenn es regnet. Denn wenn ich mich nicht freue, regnet es auch." Statt sich also mit was man als hilfreich empfinden würde - auch wenn es nicht erfüllt werden kann. Man weiß mittlerweile, dass sich das stressgeplagte Druckerleben von "Ich sollte“ auf unser Erbgut auswirken kann. Es bilden sich sogenannte Histone, die man sich wie Masken auf dem Erbgut vorstellen kann. In Momenten der Ruhe oder der achtsamen und gütigen Kommunikation mit sich werden die Genome wieder demaskiert und können ihre Arbeit im ursprünglich gedachten Sinne wieder aufnehmen. Wenn Sie also schon die Welt nicht ändern können, dann gestalten Sie den Blick auf die Welt etwas freundlicher. Damit geben Sie dem Lästern oder Abwerten weniger Raum,
Klagen und Lamentieren abzugeben, könnte man einen Schirm mitnehmen und das Beste daraus machen. Stress entsteht immer, wenn ich möchte, dass die Welt anders ist, als sie nun mal ist: Ich sollte dünner sein, mein Partner liebevoller, meine Kollegin stiller und der Stau weg. Mit systemischer Sicht betrachtet man die Welt so, wie sie sich einem jetzt zeigt. Man lenkt den Blick auf das, was ist, verliert aber die Sehnsuchtsziele nicht aus dem Auge. Man belegt die Jetzt-Situation nicht mit Abwertung, sondern behandelt sie achtsam, weil das, was sich jetzt zeigt, die jetzt bestmögliche Lösung ist.
Zeit also, die Sicht auf die Dinge zu erweitern, indem Sie zum Beispiel jeden Morgen drei Dinge finden, die Sie auf dem Weg zur Arbeit als schön, angenehm oder inspirierend betrachten. Und setzen Sie in jedem Feierabend Ihr tägliches Wertschätzungstraining fort: Was ist Ihnen heute geglückt? Wem könnten Sie dankbar sein, wer hat Ihnen heute zum Beispiel ein nettes Lächeln geschenkt? Bereits nach zwei Wochen führt diese Übung zu nachweislichen Auswirkungen auf die Aktivierung des Frontallappens und auf die Senkung des Blutdrucks. Eckart von Hirschhausen hat mal so nett gesagt: „Dann ist Ihr Frontallappen kein Jammerlappen mehr.“ Karin Probst

$\boldsymbol{\theta}$ Literaturverzeichnis

www.thieme-connect.de/products/ergopraxis $>$ „Ausgabe 9/16“

$\rightarrow$ Internet

www.youtube.com/watch?v=9TI7Wta8m4w

\section{H Gewinnen}

\section{Stressreduktion}

Wir verlosen drei Mal das Buch „Achtsamkeitsbasierte Therapie und Stressreduktion MBCT/MBSR“ vom Reinhardt Verlag (www. reinhardt-verlag.de). Wer gewinnen will, klickt bis zum 30.9.2016 unter www.thieme.de/ ergopraxis > "Gewinnspiel“ auf das Stichwort "Stressreduktion“. Viel Glück!

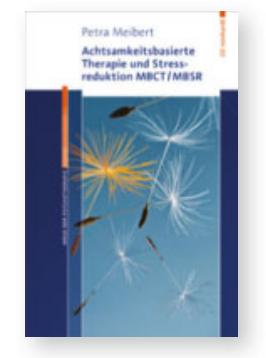

\section{Autorin}

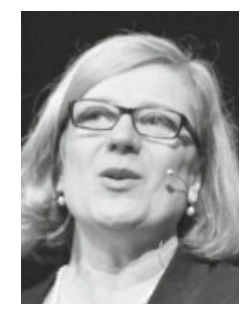

Karin Probst, zertifizierter systemischer Business Coach FU Berlin, war Schauspielerin und Regisseurin an führenden Theatern in Deutschland und begleitet seit 1998 internationale Unternehmen mit Training, Coaching und Beratung. Infos unter: www.2-change.de 